\title{
Anti-Windup and 2DOF Design for Permanent-Magnet Synchronous Motor Speed Controller
}

\author{
Xiaofan Wang, Fei Lin*, Zhongping Yang and Xiaochun Fang \\ School of Electrical Engineering, Beijing Jiaotong University, Beijing 100044, \\ China \\ flin@bjtu.edu.cn
}

\begin{abstract}
A novel speed controller for permanent-magnet synchronous motor (PMSM) is proposed. With the usage of an analytical two-degree-of-freedom (2DOF) proportional integral (PI) controller design method in speed control, good speed-reference tracking and good load-torque rejection can be achieved simultaneously. A "back calculation and tracking" anti-windup (AW) method is used to deal with the "windup" problems. In both simulation and experiment, the proposed designs are shown to exhibit satisfactory performance.
\end{abstract}

Keywords: Permanent-magnet synchronous motor (PMSM), Speed control, Twodegree-of-freedom (2DOF), Anti-windup (AW)

\section{Introduction}

Permanent-magnet synchronous motor (PMSM) is widely applied in various fields due to its advantages of high efficiency and high power density [1]. For a long time, significant attention is being directed to speed control of PMSM drives. The reasons for this interest are that the dynamics may be nonlinear and/or time varying and that it is essential to gain both good speed-reference tracking and good load-torque rejection performance. The latter two objectives cannot both be met with a standard one-degree-offreedom (1DOF) proportional integral (PI) controller. Besides, within PMSM control systems, certain constrains are required to protect the motor and the power electronics converter; for instance, restrictions on the magnitude of voltages and currents. These constrains can cause controller "windup" problems when the controller attempts to exceed them and lead to performance degradation. For better performance, there is the requirement to consider these saturation constrains in the design of PMSM control systems, particularly in high-performance applications where saturation is more prevalent.

In view of the limitations of standard 1DOF PI controller, various nonlinear methods, including neutral networks, fuzzy logic [2], and sliding mode control, have been proposed. But for the engineering application, most of these methods are too complex. Many publications focus on two-degree-of-freedom (2DOF) PI controller, [3]-[6]. The socalled 2DOF controller is not a fixed pattern, but includes a variety of forms. The basic pattern is the so-called integral proportional (IP) control [3]. Alternative methods include the usage of a phase-locked loop, auto-disturbance rejection, load-torque estimation and feedforward, and adaptive techniques. An analytical 2DOF PI controller design method was proposed in reference [4]. Unlike many of the previously proposed linear controllers, the gains of the controller are analytically parametrized in the inertia as well as in the desired closed-loop bandwidth. But the electrical dynamics is neglected in the design process.

${ }^{*}$ Corresponding Author 
To deal with the "windup" problems caused by saturation constrains, many antiwindup (AW) methods are proposed. The AW compensator variants include an integrator reset scheme, back calculation and tracking [7], and an optimally synthesized low-order dynamic compensator [8]. Performance of these three AW structures is compared through simulation and experiments in reference [9]. Both of "back calculation and tracking" and "low-order dynamic" AW compensation methods provide an improvement over the reset approach and show very similar performance in simulation despite their differing design routines and implementations. The "back calculation and tracking" approach which is applied widely in electrical drive systems is very simple to implement and easy to tune.

In this paper, we apply 2DOF design method and AW compensation method to a PMSM speed control system. Simulations and experiments will be presented to show the performance of the proposed speed controller.

\section{Model of PMSM Speed Control System}

\subsection{PMSM Model}

In dynamic analysis of PMSM, Clark and Park transformations are used to convert the three phase system ( $a b c$-axis) into two phase rotating system ( $d$-q-axis) generally. The dynamic equations of PMSM in $d-q$-axis are as follows [10], [11]:

$$
\left\{\begin{array}{l}
u_{\mathrm{d}}=R_{\mathrm{s}} i_{\mathrm{d}}+L_{\mathrm{d}} \frac{\mathrm{d} i_{\mathrm{d}}}{\mathrm{d} t}-\omega_{\mathrm{r}} i_{\mathrm{q}} L_{\mathrm{q}} \\
u_{\mathrm{q}}=R_{\mathrm{s}} i_{\mathrm{q}}+L_{\mathrm{q}} \frac{\mathrm{d} i_{\mathrm{q}}}{\mathrm{d} t}+\omega_{\mathrm{r}} i_{\mathrm{d}} L_{\mathrm{d}}+\omega_{\mathrm{r}} \psi_{\mathrm{f}} \\
T_{\mathrm{e}}=p_{0}\left[\psi_{\mathrm{f}} i_{\mathrm{q}}+\left(L_{\mathrm{d}}-L_{\mathrm{q}}\right) i_{\mathrm{d}} i_{\mathrm{q}}\right] \\
\frac{1}{p_{0}} \frac{\mathrm{d} \omega_{\mathrm{r}}}{\mathrm{d} t}=\frac{1}{J}\left(T_{\mathrm{e}}-T_{1}\right)
\end{array}\right.
$$

Where $u_{\mathrm{d}}$ and $u_{\mathrm{q}}$ are stator voltages in $d$ - $q$-axis, $i_{\mathrm{d}}$ and $i_{\mathrm{q}}$ are stator currents in $d$ - $q$-axis, $L_{\mathrm{d}}$ and $L_{\mathrm{q}}$ are inductances in $d$-q-axis, $\omega_{\mathrm{r}}$ is the rotor electrical angular speed, $R$ is the stator resistance, $\Psi_{\mathrm{f}}$ is the rotor flux, $p_{0}$ is the number of poles, $J$ is the inertia of the motor, $T_{\mathrm{e}}$ and $T_{1}$ are the electromagnetic torque and the load torque.

\subsection{Control System of PMSM}

The three phase PMSM speed control system which we desire to apply 2DOF design and AW compensation to is shown in Figure 1. The control system consists of an inner current loop and an outer speed control loop. The inner loop contains two independent PI regulators, used to control the magnitude of the stator current components at $d$ - $q$-axis, namely $i_{\mathrm{dq}}\left(i_{\mathrm{d}}\right.$ and $\left.i_{\mathrm{q}}\right)$. The speed controller is shown in Figure 2. It generates the d-axis and q-axis current commands, namely $i_{\mathrm{dq} \_ \text {cmd }}\left(i_{\mathrm{d}_{\mathrm{c}} \mathrm{cmd}}\right.$ and $\left.i_{\mathrm{q}-\mathrm{cmd}}\right)$, and passes them to the inner loop. Saturation function Sat\|l\| sets the maximum limit of $i_{\mathrm{dq} \_ \text {cmd. }}$. For maximum torque per ampere (MTPA) [12] operation, $i_{\mathrm{d}_{-} \text {cmd }}$ is calculated by $i_{\mathrm{q}-\mathrm{cmd}}$ and $\omega_{\mathrm{r}}$, particularly $i_{\mathrm{d}_{-} \text {cmd }}=0$ for surface mounted PMSM.

The transfer function of the mechanical dynamics of the PMSM is immediately found by taking the Laplace transform of (1). It is simply an integrator

$$
G_{\mathrm{m}}(s)=\frac{1}{s J}
$$

For the sake of simplicity, the current loop is equivalent to a first order inertia link [12], so the transfer function of the inner loop is

$$
G_{\mathrm{ci}}(s)=\frac{1}{\tau_{\mathrm{i}} s+1}
$$


Where $\tau_{\mathrm{i}}$ is the time constant of the electrical dynamics, which is usually tens of milliseconds.

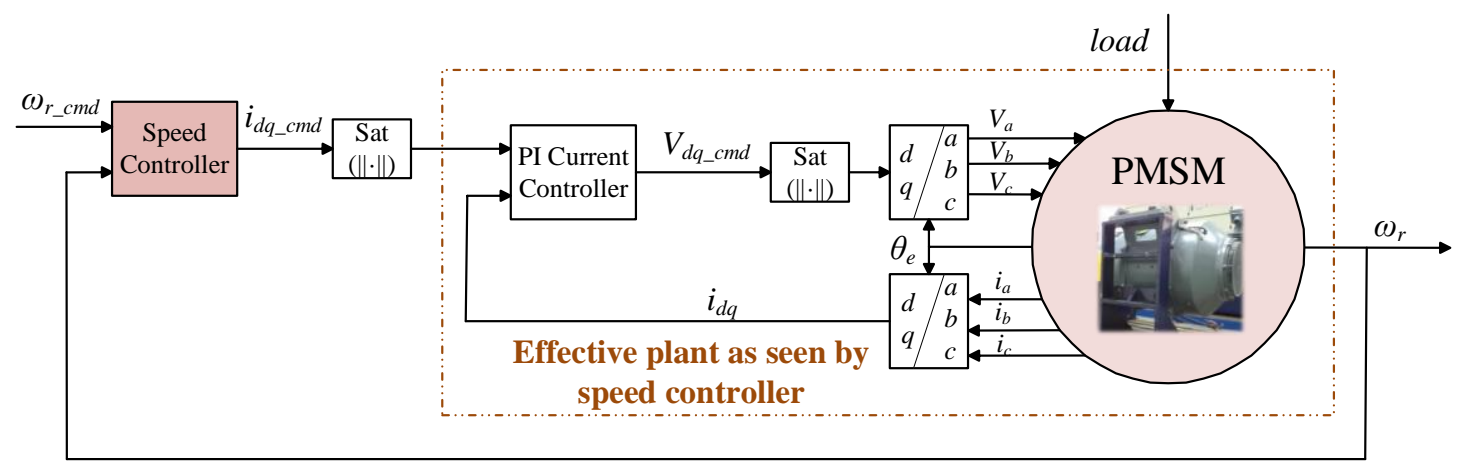

Figure 1. Speed Control System of PMSM

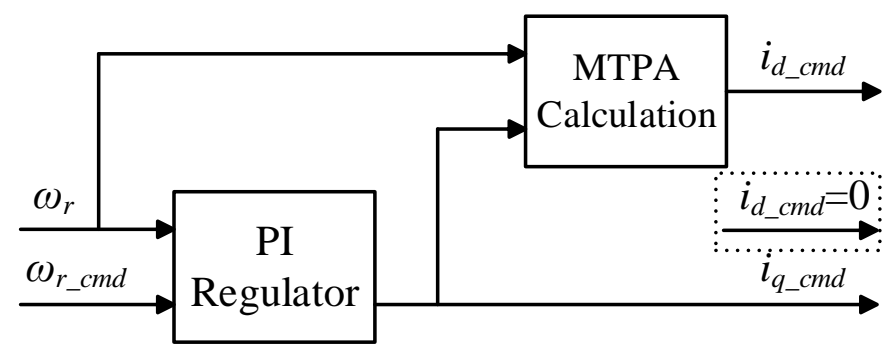

Figure 2. Speed Controller Structure

\section{Speed Controller Design}

In this section, we introduce an analytical 2DOF PI controller design method and AW compensation method and apply them to the speed controller design for PMSM.

\subsection{DOF Method}

First of all, we analyze the characteristics of the traditional control structure. Block diagram of the standard 1DOF control [3] is shown in Figure 3. Where $X(s)$ is the input, $Y(s)$ is the output, $N(s)$ is the disturbance, $G(s)$ is the controlled object and $F(\mathrm{~s})$ is the controller. The close-loop transfer function from $X(s)$ to $Y(s)$ and form $N(s)$ to $Y(s)$ are shown in (4) and (5).

$$
\begin{gathered}
G_{\mathrm{X}}(s)=\frac{F(s) G(s)}{1+F(s) G(s)} \\
G_{\mathrm{N}}(s)=-\frac{G(s)}{1+F(s) G(s)}
\end{gathered}
$$

From (4) and (5) we can see that $G_{\mathrm{X}}(s)$ and $G_{\mathrm{N}}(s)$ are both determined by $F(s)$ only. So systems with 1DOF control cannot adjust the reference tracking and disturbance rejection performance separately. 


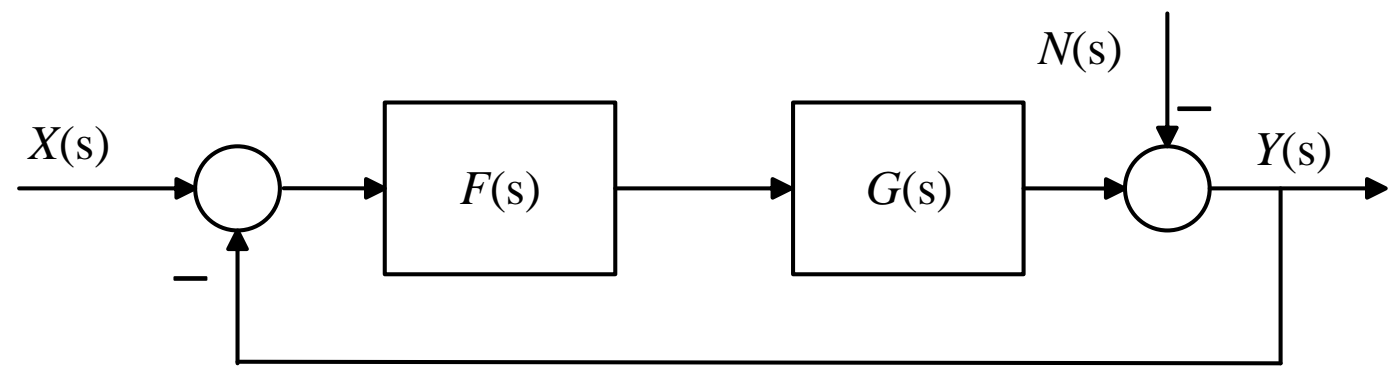

Figure 3. Block Diagram of the 1DOF Control

To make an enhancement of 1DOF control, the structure can be modified by adding a feedforward controller $F \mathrm{r}(\mathrm{s})$ for the reference, as shown in Figure 4

The close-loop transfer function from $X(s)$ to $Y(s)$ is shown in (6) and the close loop transfer function from $N(s)$ to $Y(s)$ is as same as (5).

$$
G_{\mathrm{X}}(s)=\frac{F_{\mathrm{r}}(s) G(s)+F(s) G(s)}{1+F(s) G(s)}
$$

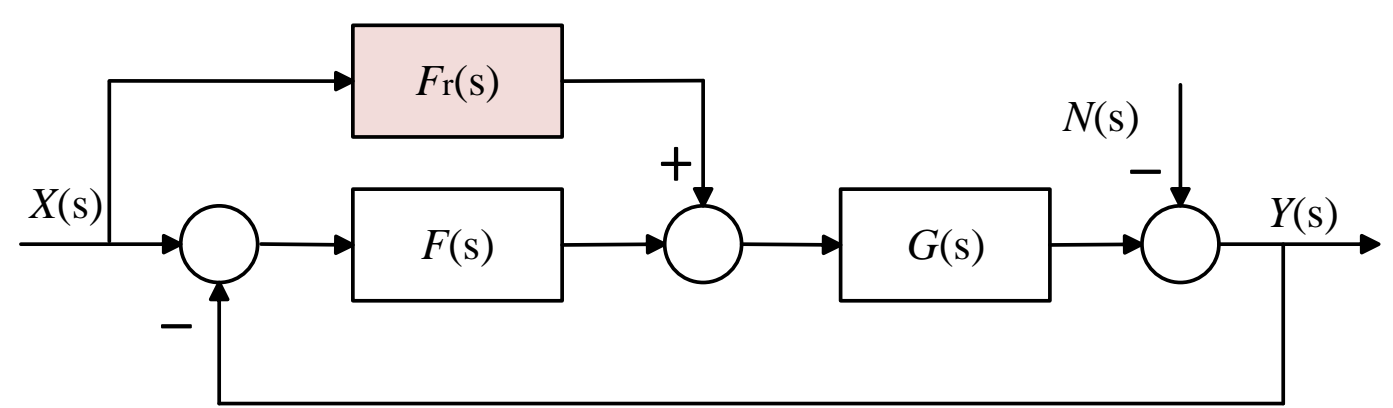

Figure 4. Block Diagram of the 2DOF Control

From (6) and (5) we can see that $G_{\mathrm{N}}(s)$ is determined by $F(s)$ only. While $G_{\mathrm{X}}(s)$ is determined by $F(s)$ and $F_{\mathrm{r}}(s)$ collectively. We can get desired disturbance rejection performance by modifying $F(s)$. And the feedforward controller $F_{\mathrm{r}}(s)$ is act as a patch to improve the reference tracking performance. When the form of $G(s)$ is identified as a goal, the transfer function of $F_{\mathrm{r}}(s)$ can be derived from (6)

$$
F_{\mathrm{r}}(s)=F(s)\left(G_{\mathrm{X}}(s)-1\right)+\frac{G_{\mathrm{X}}(s)}{G(s)}
$$

\subsection{AW Compensation}

A signal $u^{*}$ is defined as the difference of between saturated and unsaturated control signals. And $u^{*}=0$ indicates the normal operation where $u^{*} \neq 0$ indicates the existence of a saturation event. In the "back calculation and tracking" method, the AW compensator adopts the form of a scalar feedback gain $K_{\mathrm{a}}$, which is introduced between the signal $u^{*}$ and the input to the integrator in the PI controller, as is shown in Figure 5. 


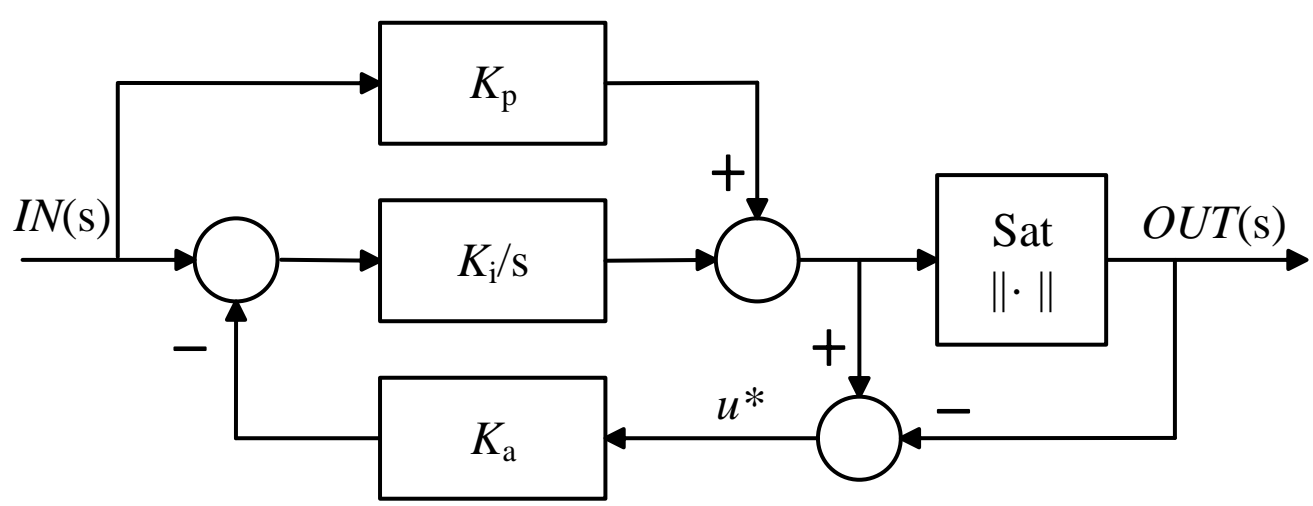

Figure 5. Block Diagram of the AW Compensation

According to engineering experience, the feedback gain $K$ a take for $1 / K_{\mathrm{p}}$ generally, can be variable in the range of $K_{\mathrm{p}} / 3 \sim 3 K_{\mathrm{p}}[11]$.

\subsection{Speed Controller Design for PMSM}

The effective plant as seen by speed controller is consisted by (2) and (3). A speed controller with excellent performance can be synthesized from 2DOF PI controller and AW compensation. The proposed speed control system of PMSM is shown in Figure 6.

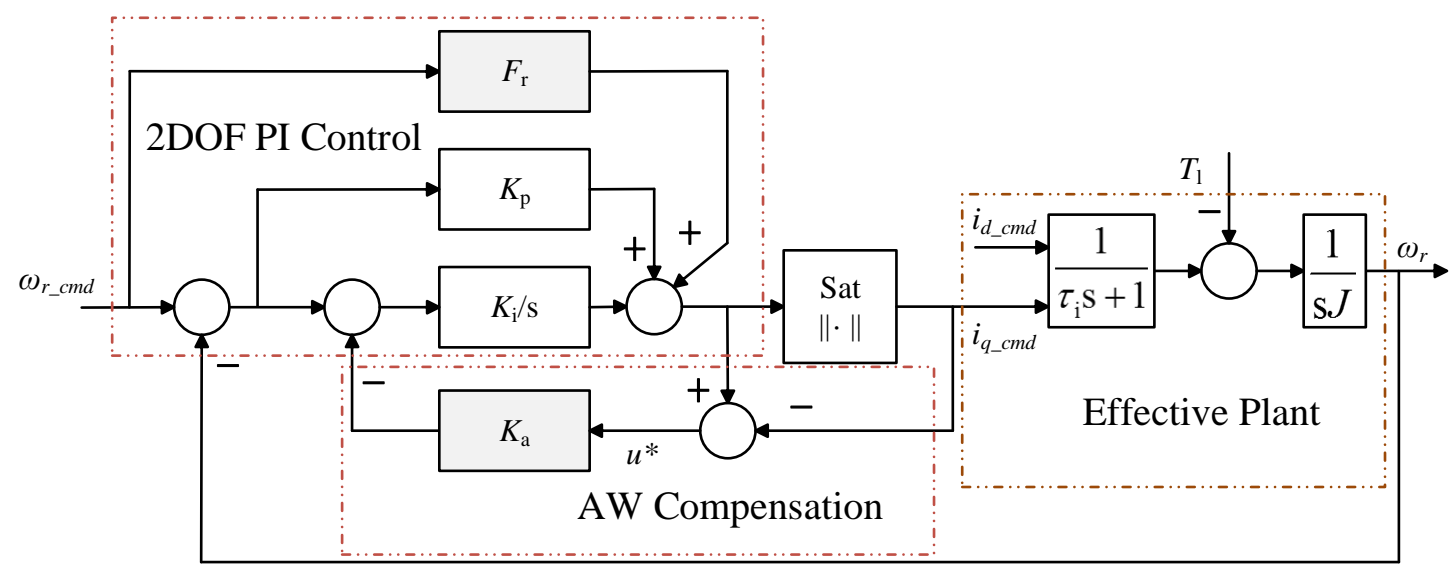

Figure 6. The Proposed Speed Control System of PMSM

In normal operation, the close-loop transfer function from $T_{1}$ to $\omega_{\mathrm{r}}$ is

$$
G_{\mathrm{N}}(s)=-\frac{\tau_{\mathrm{i}} s^{2}+s}{J \tau_{\mathrm{i}} s^{3}+J s^{2}+K_{\mathrm{p}} s+K_{\mathrm{i}}}
$$

Parameter selection of the PI controller is count for much to the transient load-torque rejection. We chose $K_{\mathrm{p}}$ and $K_{\mathrm{i}}$ as follows

$$
\left\{\begin{array}{l}
K_{\mathrm{p}}=J \alpha \\
K_{\mathrm{i}}=J\left(\frac{\alpha}{2 \zeta}\right)^{2}
\end{array}\right.
$$

Where $\alpha$ is the bandwidth of the controller in pure $\mathrm{P}$ control, $\zeta$ is the relative damping in standard PI control [4].

To decrease the overshot appearing in the step response of the standard 1DOF PI control, first-order dynamics can be selected as the desired transfer function of $G_{\mathrm{X}}(s)$ 


$$
G_{\mathrm{X}}(s)=\frac{m \alpha}{s+m \alpha}
$$

$G_{\mathrm{X}}(s)$ has a pole at $s=-m \alpha$ and parameter $m$ can be used to freely select the location of the pole. Then the feedforward controller can be obtained by (2), (3), (7), (9) and (10)

$$
F_{\mathrm{r}}(s)=J \alpha \frac{m \tau_{\mathrm{i}} s^{2}+(m-1) s-\frac{\alpha}{4 \zeta^{2}}}{s+m \alpha}
$$

The influence of parameter $m$ to the close-loop system is shown in Figure 7 by bode diagram (with $\alpha=0.5$ and $m$ varies from 0.2 to 2 ). From the bode diagram of $G_{\mathrm{X}}(\mathrm{s})$ we can see that increases as the parameter $m$, the bandwidth of the system increases.

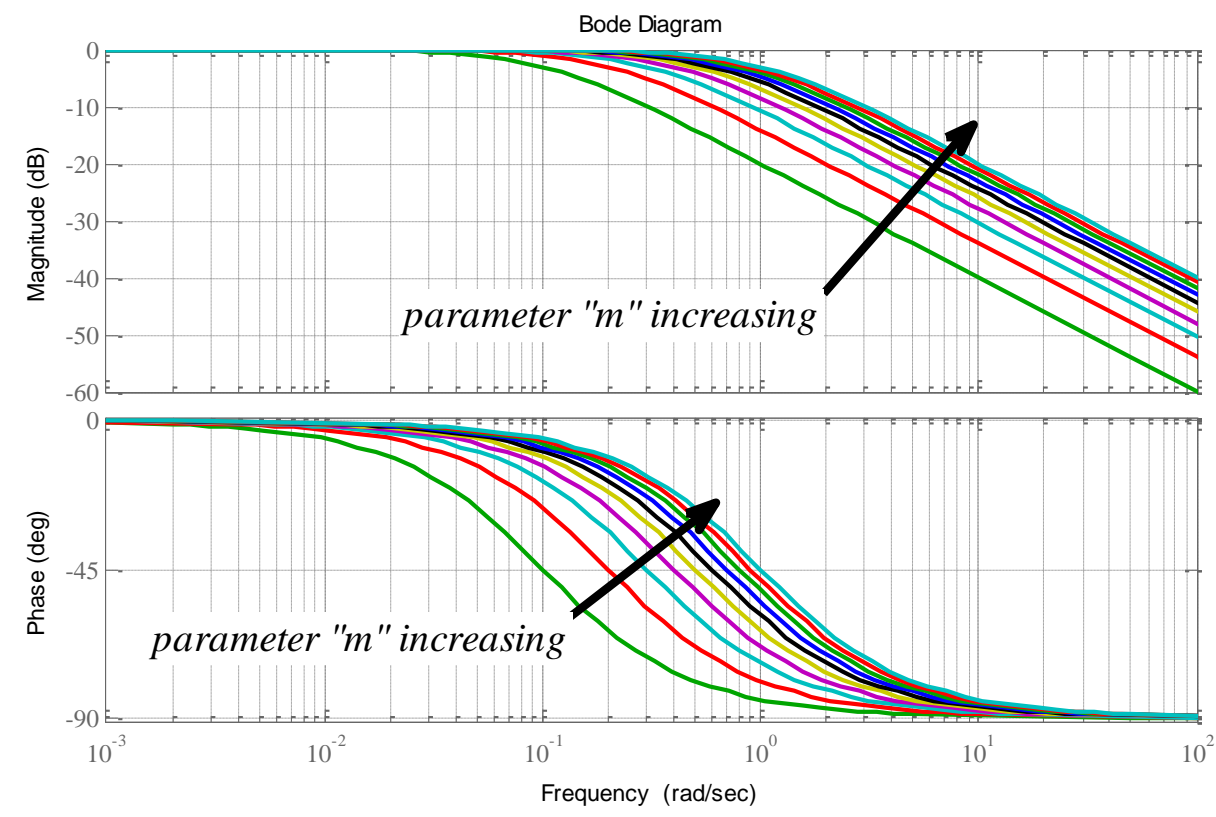

Figure 7. Bode Diagram of $G_{X}(s)$ [with $\alpha=0.5$ and $m$ Varies from 0.2 to 2]

A special case is $m=1$, then

$$
F_{\mathrm{r}}(s)=J \alpha \frac{\tau_{\mathrm{i}} s^{2}-\frac{\alpha}{4 \zeta^{2}}}{s+\alpha}
$$

It can be further simplified in case of $\tau_{\mathrm{i}}$ is very small, becomes simply a first-order lowpass filter

$$
F_{\mathrm{r}}(s)=-\frac{J\left(\frac{\alpha}{2 \zeta}\right)^{2}}{s+\alpha}
$$

When a saturation event is occurs $\left(u^{*} \neq 0\right)$, the AW compensator will take action. Then the signal $u^{*}$ will soon be reduced to zero. We choose $K_{\mathrm{a}}=1 / K_{\mathrm{p}}$ in preliminary design.

\subsection{Digital Implementation of the Controller}

The proposed speed controller can be implemented digitally on a DSP. Common discretization methods are summarized in Table ta1. 
Table 1. Discretization Methods and Transfer Relations

\begin{tabular}{c|c|c}
\hline Forward Euler & Backward Euler & Trapezoidal(Bilinear) \\
\hline$s=\left(z^{-1}\right) / T_{\mathrm{s}}$ & $s=\left(z^{-1}\right) /\left(z T_{\mathrm{s}}\right)$ & $s=\left[2\left(z^{-1}\right)\right] /\left[T_{\mathrm{s}}(z+1)\right]$ \\
\hline
\end{tabular}

Where $T_{\mathrm{s}}$ is the sampling period. Among these methods, Trapezoidal method usually has better performance. With the usage of the transfer relation of Trapezoidal method, the transfer functions of the PI controller and the feedforward controller will become

$$
\begin{gathered}
F(z)=K_{\mathrm{p}}+K_{\mathrm{i}} \frac{T_{\mathrm{s}}}{2} \frac{1+z^{-1}}{1-z^{-1}} \\
F_{\mathrm{r}}(z)=-\frac{J\left(\frac{\alpha}{2 \zeta}\right)^{2}}{\frac{2}{T_{\mathrm{s}}} \frac{1-z^{-1}}{1+z^{-1}}+\alpha}
\end{gathered}
$$

\section{Simulations and Experiments}

\subsection{Simulations}

For simulation tests, the control system of PMSM which is shown in Figure 1 is established in MATLAB/Simulink. Then we compare the performance of standard speed controller and the proposed speed controller. The inertia of the motor used in simulation is $J=0.4 \mathrm{~kg} \cdot \mathrm{m}^{2}$, the time constant of the inner loop is about $\tau_{\mathrm{i}}=0.05 \mathrm{~s}$, the limit of $i_{\mathrm{q}_{\mathrm{C}} \mathrm{cmd}}$ is 7.6A.

Simulation condition 1: with no load, speed reference $500 \mathrm{rpm}$ is given at $t=0 \mathrm{~s}$, and $200 \mathrm{rpm}$ at $t=5 \mathrm{~s}$.

The performance of the AW compensation method is shown in Figure 8 and Figure 9 . When the step speed reference comes, $i_{\mathrm{q}_{-} \text {cmd }}$ quickly rise to the limit and hold on. Then the "windup" problem comes. Both of the speed and current responses of the control system without the AW compensation show great overshot which is more than $55 \%$ under step speed references. Furthermore, the overshot lead to the reverse impact on $i_{\mathrm{q} \_ \text {cmd }}$. The responses of the proposed controller shows better performance. The integrator quickly out of saturation so that the overshot is smaller, about $16 \%$.

Simulation condition 2: with no load, speed reference $500 \mathrm{rpm}$ is given at $t=0 \mathrm{~s}$, then load-torque $T_{\mathrm{l}}=8 \mathrm{Nm}$ is given at $t=7 \mathrm{~s}$, and speed reference $200 \mathrm{rpm}$ at $t=10 \mathrm{~s}$.

The performance of the 2DOF PI control method is shown in Figure 10. By the usage of a standard PI control method, good load-torque rejection can be met when we choose $K_{\mathrm{p}}=0.2$ and $K_{\mathrm{i}}=0.3$ (with $\alpha=0.5$ ). Then we use a feedforward controller designed according to (15), the speed-reference tracking is going to be better and good load-torque rejection still remains. 


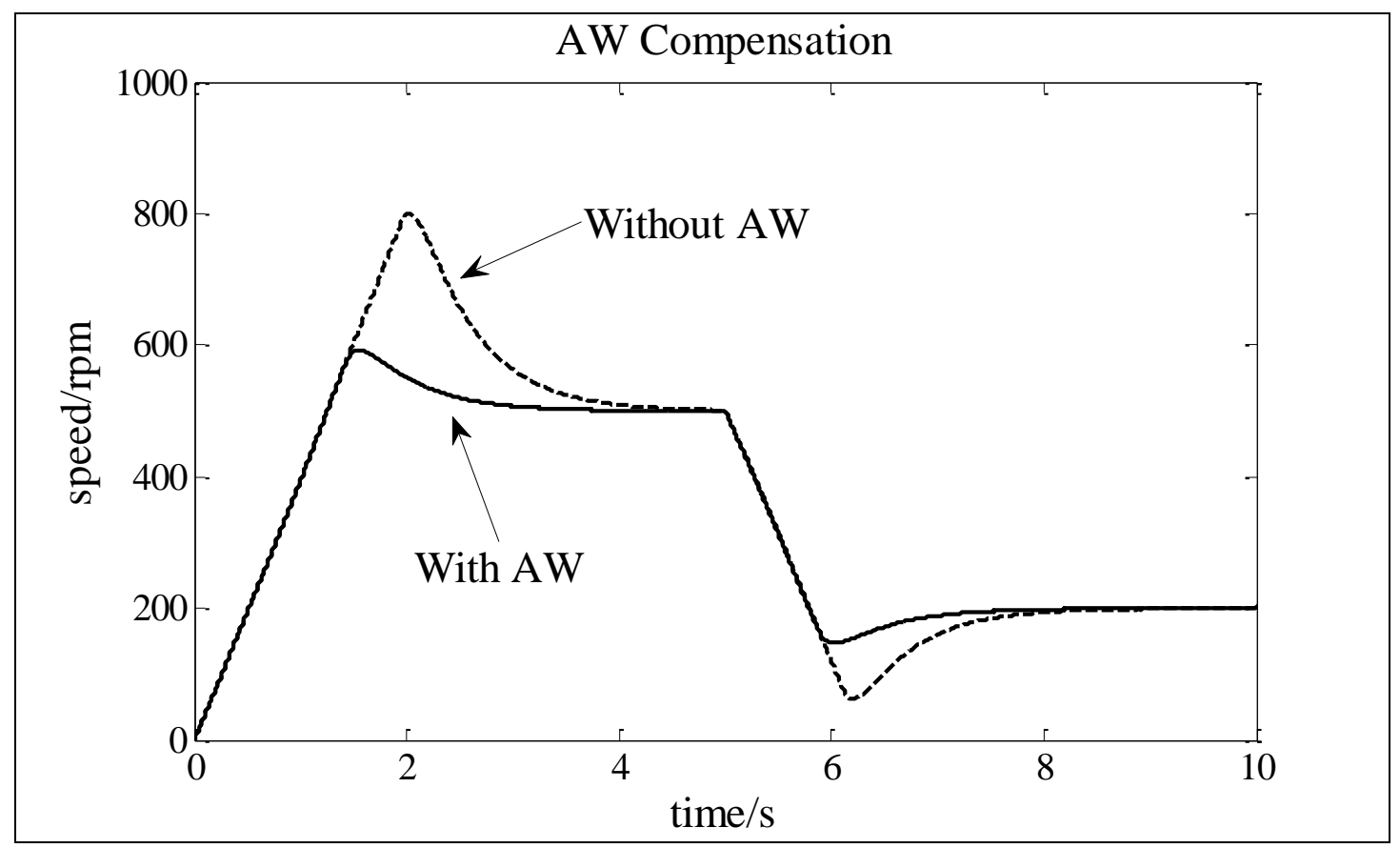

Figure 8. The Performance of the AW Compensation Method [speed]

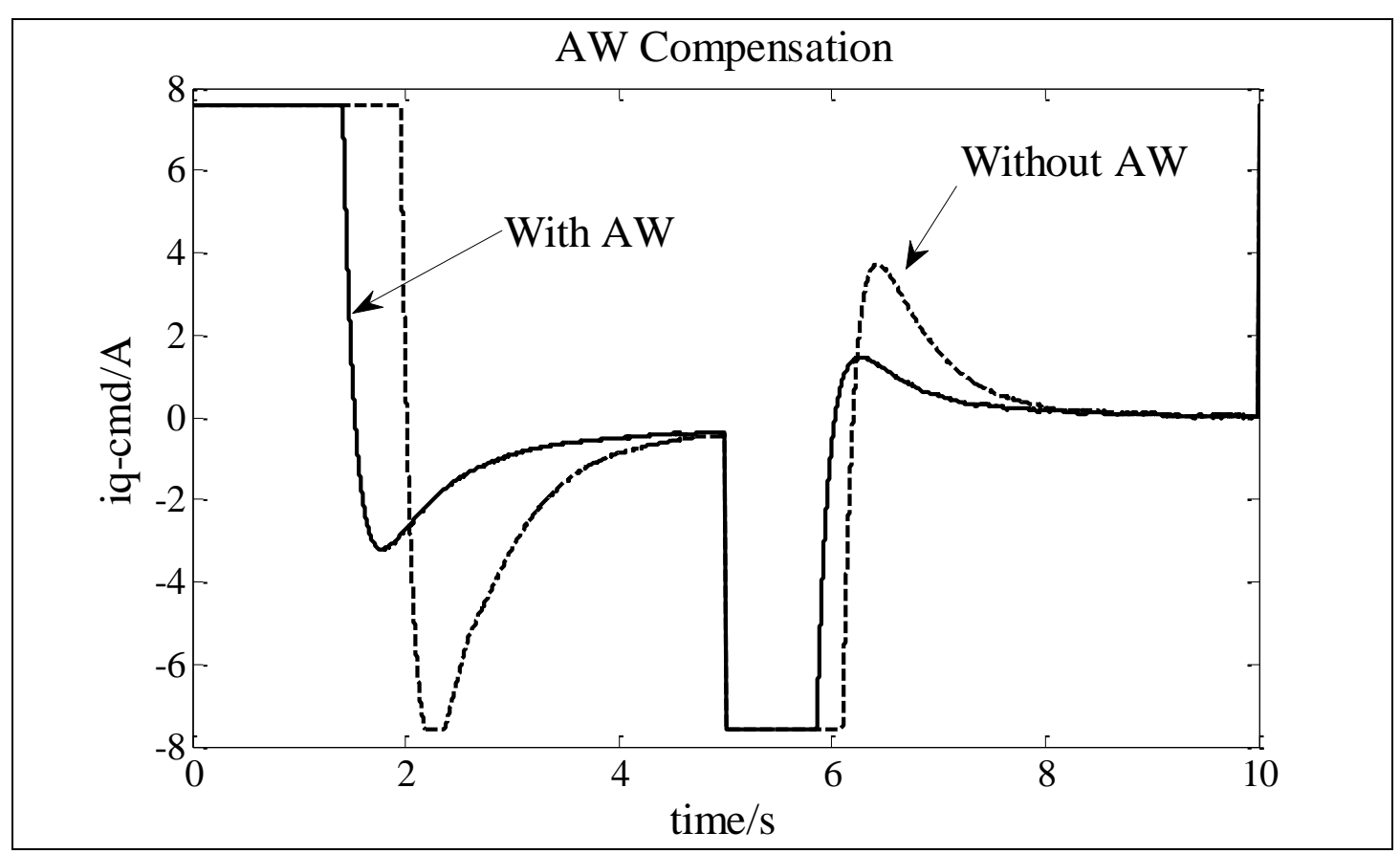

Figure 9. The Performance of the AW Compensation Method [current] 


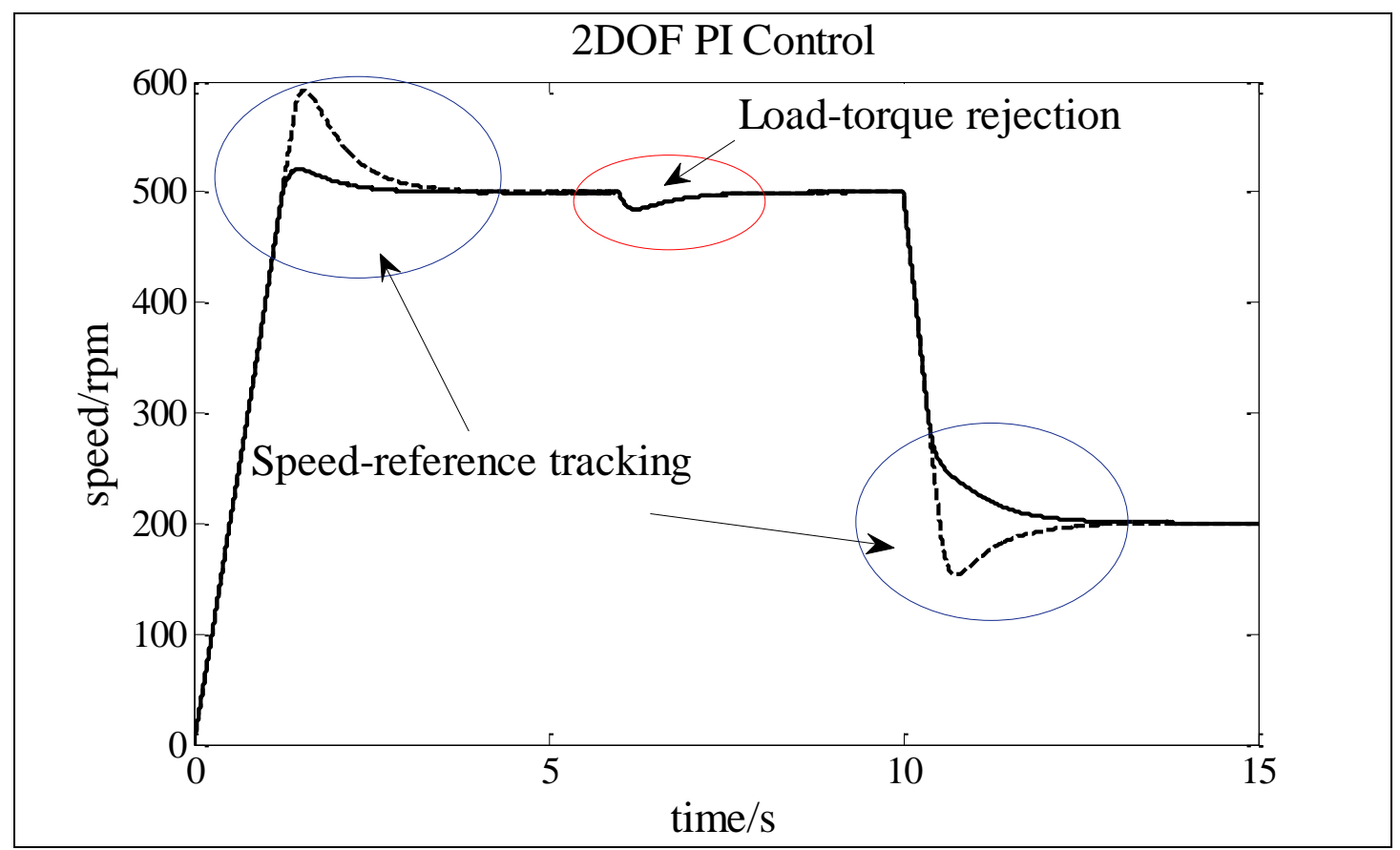

Figure 10. The Performance of the 2DOF PI Control Method

\subsection{Experiments}

For experimental tests, a drive system of PMSM which is shown in Figure 11 is established. A magnetic powder brake works as the load of PMSM. The inertia of the motor used in experiment is $J=0.4 \mathrm{~kg} \cdot \mathrm{m}^{2}$, the time constant of the inner loop is about $\tau_{\mathrm{i}}=0.05 \mathrm{~s}$, the limit of $i_{\mathrm{q}_{\mathrm{c}} \mathrm{cmd}}$ is $7.6 \mathrm{~A}$.

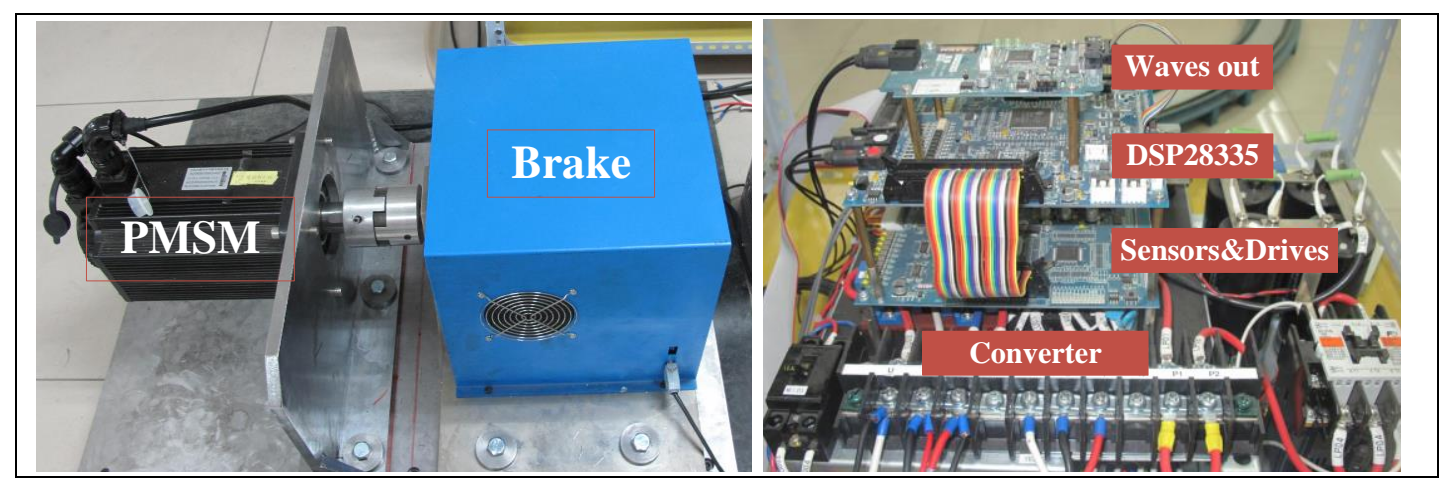

Figure 11. The Setups of the PMSM Drive System

The performance of the proposed control method is shown in Figure 12 to Figure 14.

Experimental condition 1: With no load, speed reference 500rpm is given at $\mathrm{t}=0.5 \mathrm{~s}$. The speed responses are shown in Figure 12. The response of the controller with AW compensation shows better performance. By the use of AW compensation method, the overshot is reduced from $35 \%$ to $15 \%$. 


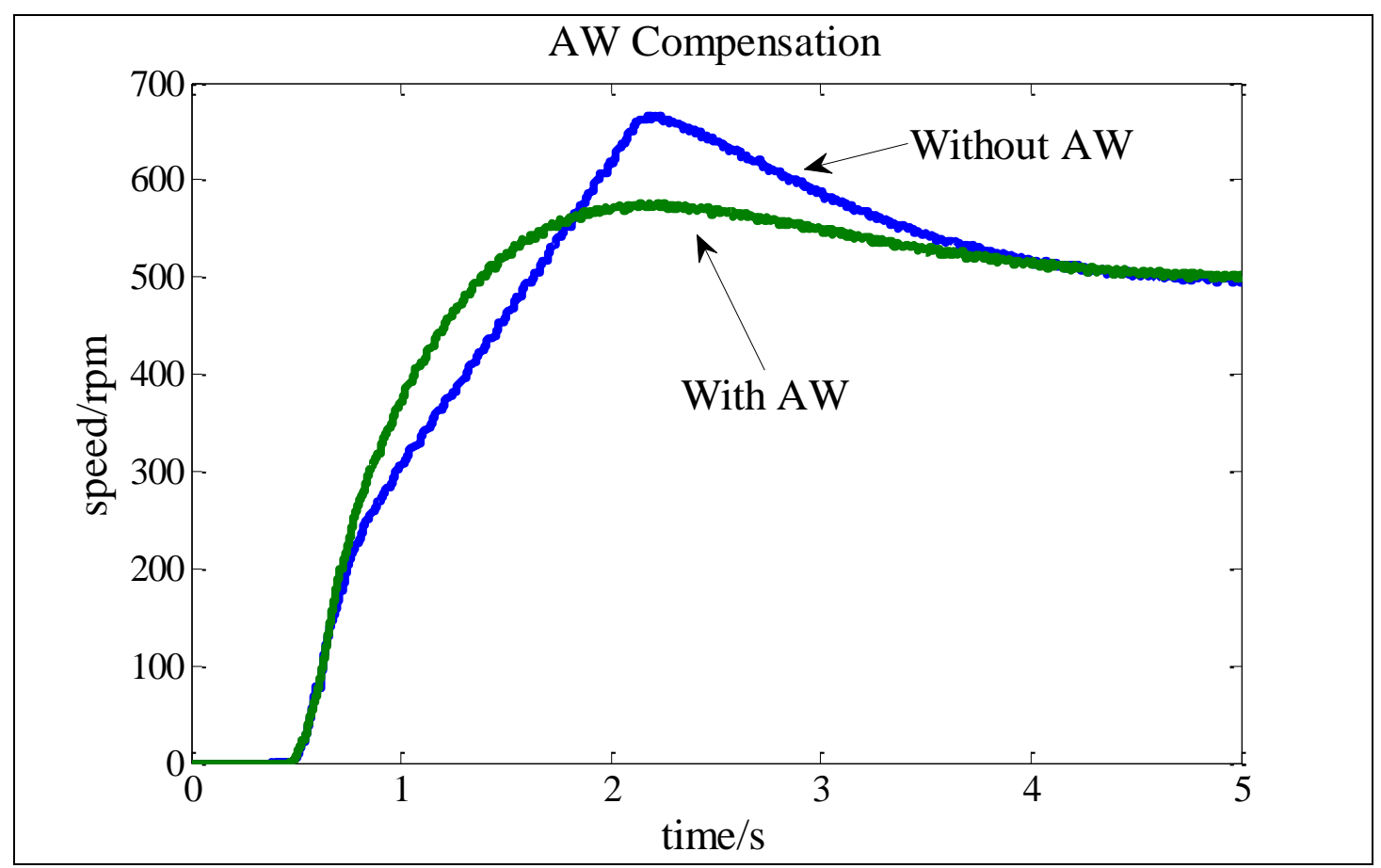

Figure 12. The Speed Responses with AW and Without AW Compensation

Experimental condition 2: With no load, speed reference step-up (from 0rpm to 500rpm) and step-down (from 500rpm to 200rpm) are given; Figure 13 shows the speedreference tracking performance of the system.

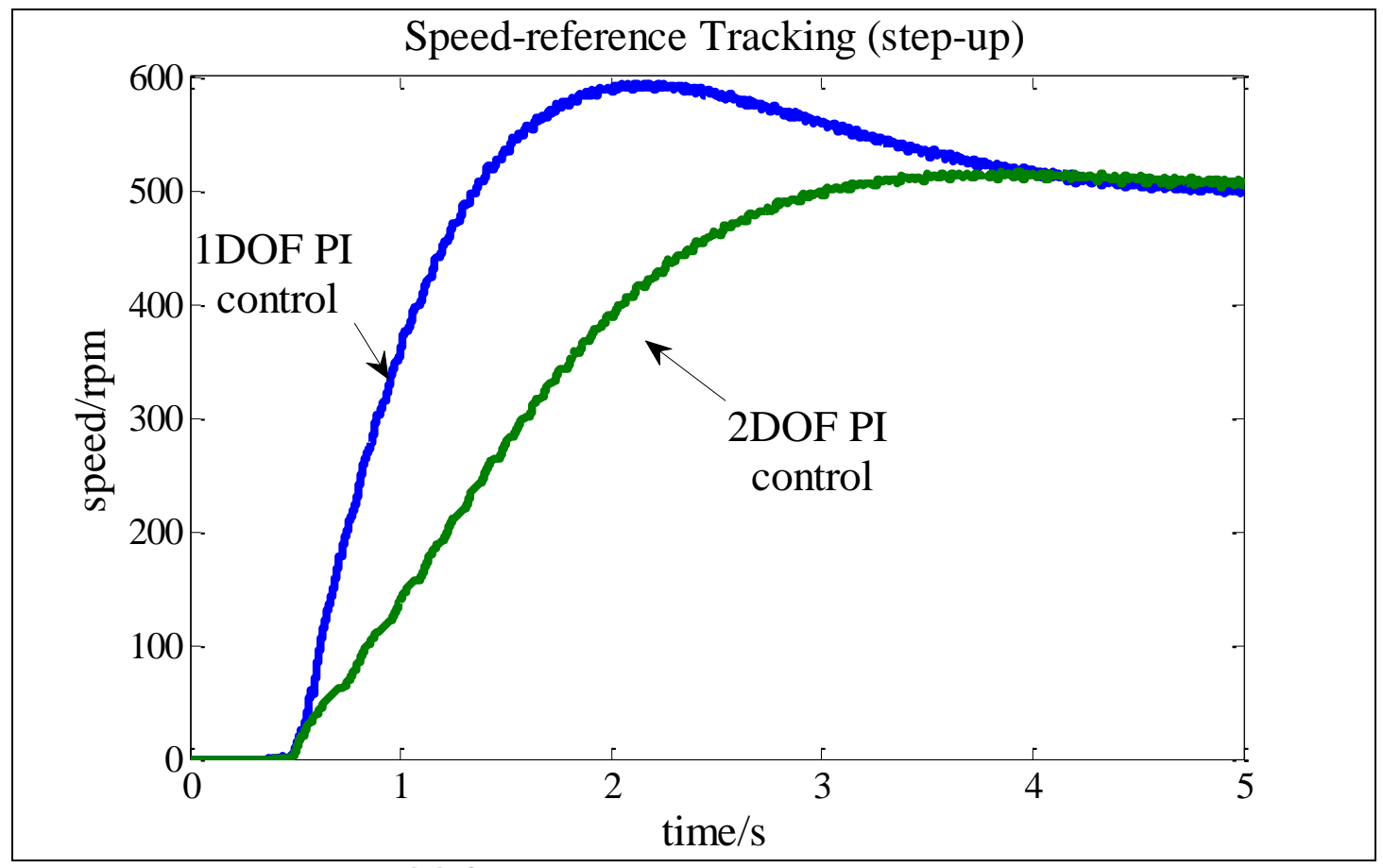

(a) Step-up of speed reference 


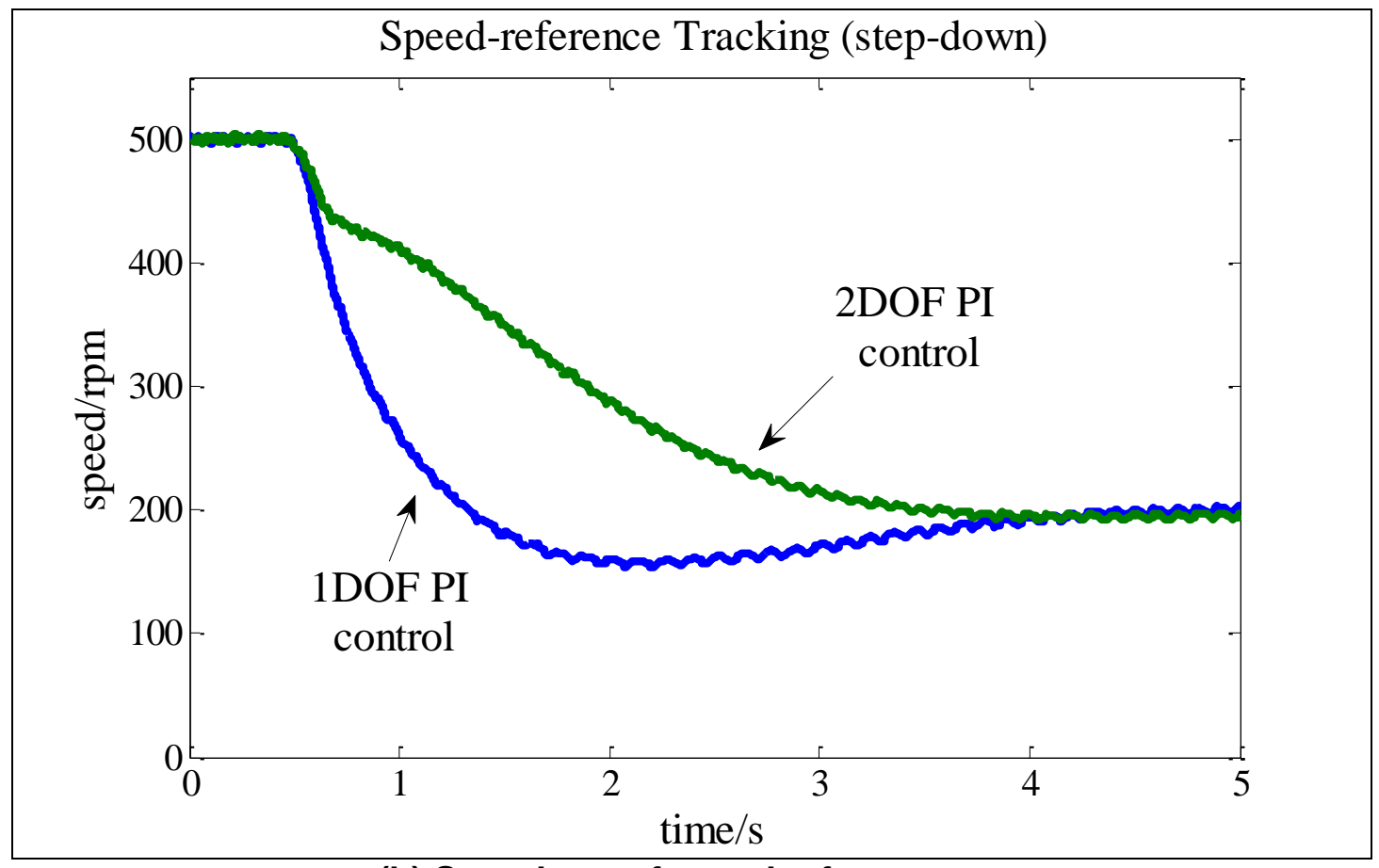

(b) Step-down of speed reference

Figure 13. The Speed-Reference Tracking Performance

Experimental condition 3: At steady operation of 500rpm, load-torque $8 \mathrm{Nm}$ (there may be a little error) is given at $t=0.5 \mathrm{~s}$. Figure 14 shows the load-torque performance of the system.

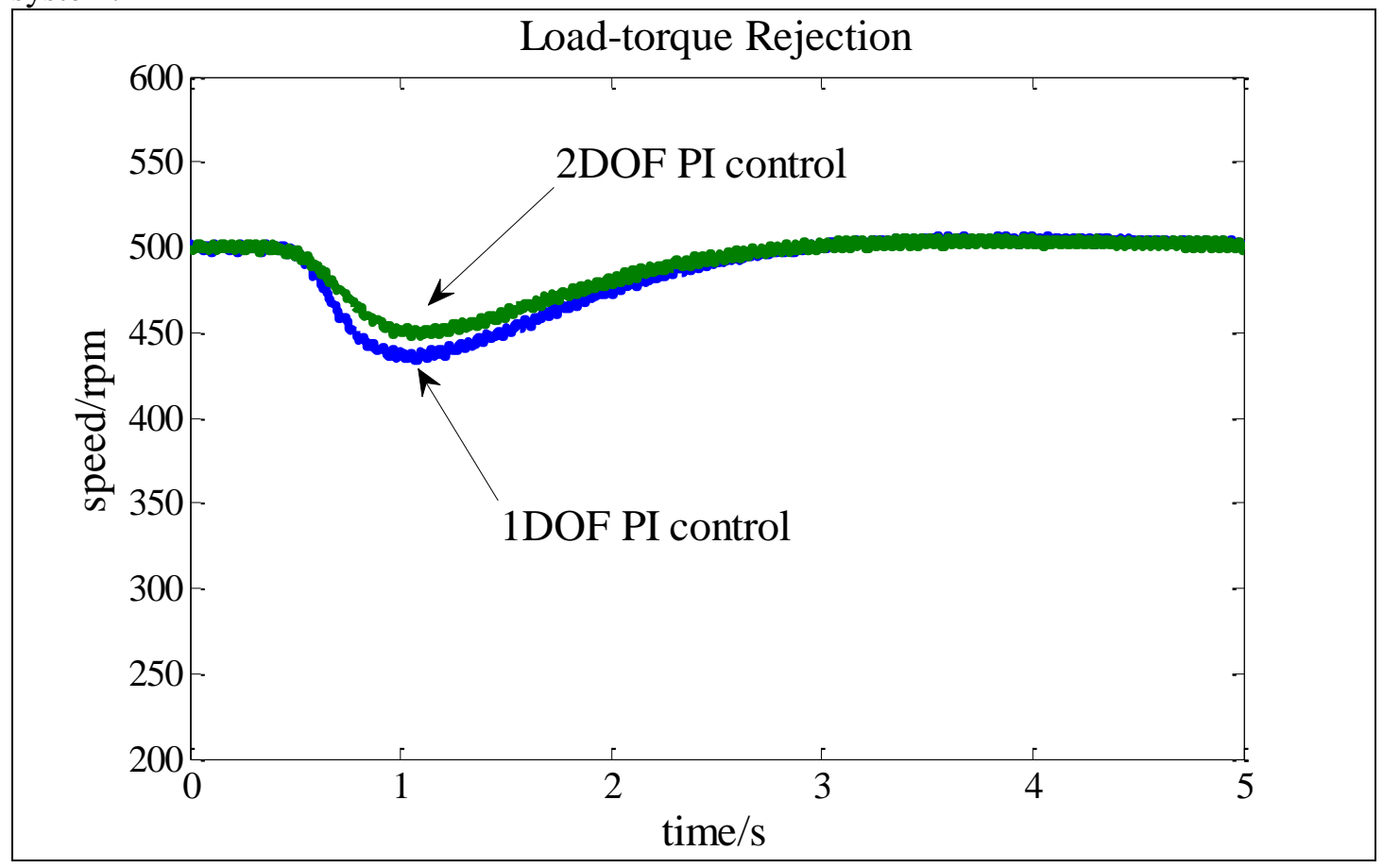

Figure 14. The Load-Torque Rejection Performance

With the usage of 2DOF PI control method, the speed-reference tacking performance has improved a lot, while the load-torque rejection is remain almost the same as in the case of the 1DOF PI control. In other words, the speed-reference 
tacking performance and the load-torque rejection performance can be adjusted separately. The experimental results shows that the proposed controller gives good reference tracking as well as good load rejection.

\section{Conclusion}

In this paper, a novel speed control method of PMSM has been proposed. The proposed controller can be easily implemented in software and needs no additional hardware or sensor. The "back calculation and tracking" AW compensation method can easily solve the "windup" problem of the controller. The 2DOF method gives the controller the ability to meet good speed-reference tracking and good load-torque rejection simultaneously. The effectiveness and superiority of the proposed method has been verified by the simulation and experimental results.

Hence, the load of the motor in real applications may not be as simple as in this paper. More advanced speed control schemes may be useful if the mechanical systems are more complex, such as a multibody system, or the nonlinearities of the model cannot be linearized. In that case, a higher order model of the load and the motor will be needed for stability analysis.

\section{Acknowledgments}

This paper is part of projects supported by a grant from the National Natural Science Foundation of China (51577010)

\section{References}

[1] H. Zang, D. Lv and Y. Dai, "Control Strategy of Permanent Magnet Synchronous Motor Used in Electric Vehicle", International Journal of Control and Automation, vol.7, no.11, (2014), pp.249-258.

[2] Z. Ibrahim and E. Levi, "A Comparative Analysis of Fuzzy Logic and PI Speed Control in HighPerformance AC Drives Using Experimental Approach", IEEE Transactions on Industry Applications, vol. 38 , no.5, (2002), pp.1210-1218.

[3] G. Saravanakumar, G. Kannan and N. P. Mahalik, "Comparative Analysis of Single and Two DOF Controllers for Integrating and Time-delay Processes", International Journal of Automation and Control, vol.7, no.1/2, (2013), pp.42-61.

[4] L. Harnefors, S. E. Saarakkala and M. Hinkkanen, "Speed Control of Electrical Drives Using Classical Control Methods", IEEE Transactions on Industry Applications, vol.49, no.2, (2013), pp.889-898.

[5] T. Nagashio, T. Kida, Y. Hamada and T. Ohtani, "Robust Two-Degrees-of-Freedom Attitude Controller Design and Flight Test Result for Engineering Test Satellite-VIII Spacecraft", IEEE Transactions on Control Systems Technology, vol.22, no.1, (2014), pp.157-168.

[6] M. Hanif, V. Khadkikar, W. Xiao and J. L. Kirtley, "Two Degrees of Freedom Active Damping Technique for LCL Filter-Based Grid Connected PV Systems", IEEE Transactions on Industrial Electronics, vol.61, no.6, (2014), pp.2795-2803.

[7] K. J. Åström and T. Hägglund, "Advanced PID control", ISA-The Instrumentation, Systems and Automation Society, Research Triangle Park, North Carolina, (2006).

[8] G. Grimm, J. Hatfield, I. Postlethwaite, A. R. Teel, M. C. Turner and L. Zaccarian, "Anti-windup for stable linear systems with input saturation: An LMI-based synthesis", IEEE Transactions on Automation Control, vol.48, no.9, (2003), pp.1509-1525.

[9] P. March and M. C. Turner, "Anti-Windup Compensator Designs for Nonsalient Permanent-Magnet Synchronous Motor Speed Regulators", IEEE Transactions on Industry Applications, vol.45, no.5, (2009), pp.1598-1609.

[10] R. Krishnan, "Electric Motor Drives, modeling, Analysis \& Control”, Prentice-Hall, Englewood, (2001).

[11] S. K. Sul, "Control of Electric Machine Drive Systems", John Wiley \& Sons, Hoboken, (2011)

[12] M. Preindl and S. Bolognani, "Optimal State Reference Computation with Constrained MTPA Criterion for PM Motor Drives”, IEEE Transactions on Power Electronics, vol.30, no.8, (2015), pp.4524-4535. 


\begin{abstract}
Authors
Xiaofan Wang, he was born in Hebei Province, China in 1992. He is currently studying for a master's degree in Electrical Engineering School in Beijing Jiaotong University. His research direction is power electronics and electrical drives.
\end{abstract}

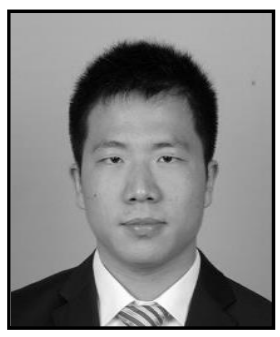

Fei Lin, he was born in Shandong Province, China in 1975. He received his B.S., M.S. and $\mathrm{Ph} . \mathrm{D}$. in Electrical Engineering from Xi'an Jiaotong University, China, Shandong University, China and Tsinghua University, China, in 1997, 2000 and 2004, respectively. Since 2004, he has been with School of Electrical Engineering, Beijing Jiaotong University, where he is currently an Associate Professor. His research interests are in the areas of power electronics and motor control. He is a member of IEEE.

Zhongping Yang, he obtained his PhD from Tokyo University in 2002 and worked in Railway vehicle technical Department in Japan Toshiba Company. Now he is a professor of Electrical Engineering School in Beijing Jiaotong University. His major researches interests are in high speed train traction drive system and energy saving technology.

Xiaochun Fang, he was born in Jiangxi Province, China in 1988. He is currently studying for a $\mathrm{PhD}$ in Electrical Engineering School in Beijing Jiaotong University. His research direction is power electronics and electrical drives. 
International Journal of Control and Automation Vol. 9, No. 12 (2016) 